\title{
The Vertical Ionosphere Parameters Inversion for High Frequency Surface Wave Radar
}

\author{
Xuguang Yang, ${ }^{1,2}$ Changjun Yu, ${ }^{3}$ Aijun Liu, ${ }^{3}$ Linwei Wang, ${ }^{3}$ and Taifan Quan ${ }^{1}$ \\ ${ }^{1}$ Department of Electronic and Information Engineering, Harbin Institute of Technology, Harbin, China \\ ${ }^{2}$ Department of Science, Harbin Engineering University, Harbin, China \\ ${ }^{3}$ Department of Information and Communication Engineering, Harbin Institute of Technology, Weihai, China
}

Correspondence should be addressed to Changjun Yu; yuchangjun@hit.edu.cn

Received 7 May 2016; Revised 1 September 2016; Accepted 13 October 2016

Academic Editor: Khalid El-Darymli

Copyright (C) 2016 Xuguang Yang et al. This is an open access article distributed under the Creative Commons Attribution License, which permits unrestricted use, distribution, and reproduction in any medium, provided the original work is properly cited.

\begin{abstract}
High Frequency Surface Wave Radar (HFSWR), which is currently applied in over-the-horizon detection of targets and sea states remote sensing, can receive a huge mass of ionospheric echoes, making it possible for the ionospheric clutter suppression to become a hot spot in research area. In this paper, from another perspective, we take the ionospheric echoes as the signal source rather than clutters, which provides the possibility of extracting information regarding the ionosphere region and explores a new application field for HFSWR. Primarily, pretreatment of threshold segmentation as well as connected region generation is used in the Range-Doppler (R-D) Spectrum to extract the ionospheric echoes. Then, electron density and plasma frequency of field aligned irregularities (FAIs) caused by plasma instabilities in the F region are obtained by the coherent backscattered radar equation. The plasma drift velocity of FAIs can also be estimated from Doppler shift. Ultimately, the effectiveness of inversion is verified by comparing with IRI2012.
\end{abstract}

\section{Introduction}

HFSWR, taking advantage of the sea-surface diffraction character of vertical polarized wave, has won great success in over-the-horizon sea target detection [1] and sea states remote sensing [2]. Ideally, a perfect conductive plane consisting of sea surface is infinite in coverage area, thus making electromagnetic wave of HFSWR completely travel along the sea. But considering the actual antenna pattern characteristics, poor ground, and array error, partial energy is radiated into sky and reflected by ionosphere. Finally the echoes arrived at radar receiver in various paths, interfering target detection severely as ionospheric clutter $[3,4]$.

Many investigations have been made on the suppression of ionospheric clutter [4-7], which have also obtained many characteristics of ionospheric clutter. Ionospheric clutter coming from different ionosphere layers has different Doppler shift, for example, Doppler shift from E layer is less than that of $\mathrm{F}$ layer. Partial ionosphere clutter has obvious directivity [8]. According to statistical results, the amplitude of most ionospheric clutter is approximate to Rayleigh distribution, while others satisfy Weibull distribution [9]. In addition, the specular clutter coming from near-vertical direction exhibits no apparent resultant directivity while the spread clutter coming from lower elevation angle has high directivity [10]. Furthermore, ionospheric clutter occupying a few range bins, such as specular scattering from Es layer or F layer, has coincident directivity, while ionospheric clutter occupying more range bins, such as from the spread $\mathrm{F}$ layer, does not have directivity. And the directivity of ionospheric clutter does not change with frequency [11].

In this paper, we take the ionosphere clutter as signal source to obtain relative parameters. The clutter signal definitely contains characteristic information of the ionosphere for its reflection from ionosphere. Currently there are two common methods for ionosphere sounding: statistical model and sounding in real-time. The widely used statistical model is the International Reference Ionosphere (IRI). For a given geography and time, the IRI can provide monthly averages of electron density, electron temperature, the molecular composition of the ions, and several additional parameters in the range of altitudes from $50 \mathrm{~km}$ to $2000 \mathrm{~km}$. The latest 
standard is IRI-2012. The advantage of IRI is that it develops as a data-based model to avoid uncertainty of theory-based models. The amplitude of these longitudinal variations is generally smaller in IRI than what is observed because IRI is based on monthly averages and the averaging process smoothes out some of structures. The disadvantage of IRI is strongly dependent on the underlying database. Regions and time periods that are not well covered by the database will result in diminished reliability of the model in these areas [12]. There are only 20-30 ionosphere stations in China; thus the IRI parameters of most regions coming from interpolation should have certain deviations from the actual measurement. Ionosonde is the widely used instrument for ionosphere sounding. Electron density profile, virtual heights, polarization, and Doppler shift can be obtained according to the ionogram of 1-30 MHz scanning-frequency results. But the limitations of the Doppler resolution from ionosonde are too low to measure the ionosphere disturbance [13]. In [13, 14], Zhou et al. tried to use HFSWR to sounding the information regarding ionosphere. More precisely, in [13], the authors obtained the time-varying ranges, virtual heights, and horizontal drifting speeds of ionosphere irregularities by using the method of time-frequency distribution and continuous hours of long time observations. In [14], the authors observed that the distance, Doppler shift, and multipath propagation of ionospheric backscatter signals changed with time. But there are few interpretations on the physical mechanism of ionospheric clutter. This paper thus tries to explain the physical mechanism between the HFSWR wave and ionosphere while estimating the electron density, plasma frequency, and drift velocity of irregularities created by plasma instabilities in the corresponding ionosphere region by the coherent backscattered radar equation.

The organization of this paper is as follows. In Section 2, the HFSWR system is introduced. In Section 3, the method to obtain the vertical ionospheric echoes from the R-D spectrum is given. In Section 4, the electron density, plasma frequency, and drift velocity of irregularities are obtained. In Section 5, experiments had proceeded at different time and were compared with IRI2012 to verify the effectiveness of the method. Conclusions are summarized in Section 6.

\section{High Frequency Surface Wave Radar System}

In this paper, measured data come from the HFSWR which is designed by Harbin Institute of Technology. The radar system transmits a frequency modulated interrupted continuous waveform with operating frequency between 3 and $15 \mathrm{MHz}$. The radar comprises an 18-element log periodic dipole transmitting antenna and an 8-element quadlet (one array element, which is formed by four vertical elements with $1 / 4$ wavelength spacing) vertically polarized antenna receiving arrays, as shown in Figures 1 and 2, respectively, at Weihai, Shandong.

Figures 3 and 4 show sketch maps of the log periodic dipole transmitting antenna and the vertically polarized antenna receiving arrays, respectively. Figure 5 shows the pattern of log periodic dipole transmitting antenna by High Frequency Structure Simulator (HFSS). It obviously shows

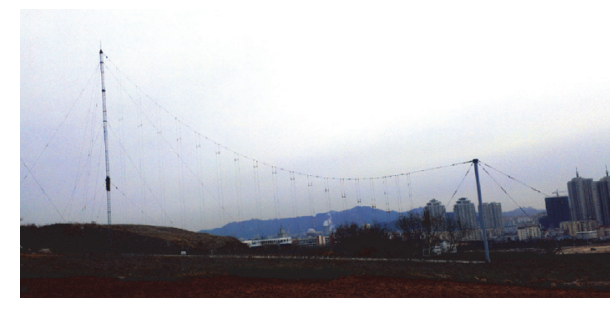

FIGURE 1: Transmitting antenna.

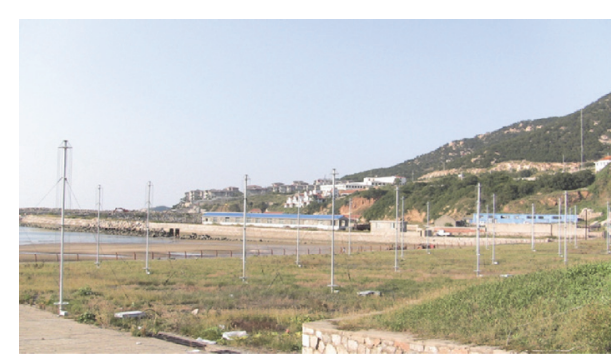

FIgURE 2: Receiving antenna arrays.

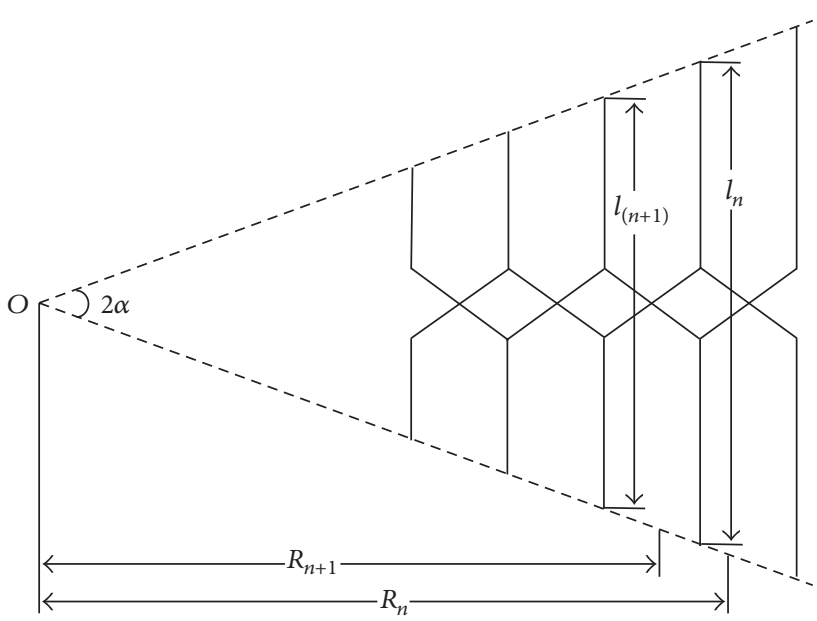

FIGURE 3: Log periodic dipole antenna.

that the antennae radiate partial electromagnetic wave to elevation especially to the zenith regardless of frequency. Figure 6 shows the pattern of receiving antenna at $9 \mathrm{MHz}$ also from HFSS. We can see that the receiving gain of zenith remains about $-8 \mathrm{~dB}$ on an ideal conductive plane. If considered on a nonideal conductive plane (the electrical parameters for the poor ground are $\sigma_{1}=0.003 \Omega^{-1} \mathrm{~m}^{-1}, \varepsilon_{r l}=$ 4 , where $\sigma_{1}$ is the conductivity and $\varepsilon_{r l}$ is relative dielectric constant of the land), the zero of vertical pattern shifts from zenith to left, whether in transmit or receive pattern [4]. So HFSWR certainly can receive the ionosphere echoes from zenith in practical engineering system.

\section{Pretreatment of Ionospheric Echoes}

Generally, HFSWR transmits frequency modulated interrupted continuous (FMICW) and receives signals by the 
uniform liner array (ULA). By using pulse compression, digital beam forming (DBF), and fast Fourier transformation (FFT) for the receiving signals from every antenna element, we can obtain the Range-Doppler (R-D) spectrums of arbitrarily azimuth angles. Since each R-D spectrum includes ionosphere echoes from all elevation angles, it is required to extract the vertical ionospheric echoes firstly.

The R-D spectrum of each beam contains the ionospheric echoes with all elevation angles, so the overlaps of all the beams only cover the elevation direction of 90 degrees. Therefore, the vertical ionospheric echoes can be obtained by extracting overlaps of all beams at the same time.

But R-D spectrum also contains sea clutter, meteoric trail, and atmospheric noise, so it should be pretreated. The pretreatment procedure includes the threshold segmentation and generation of connected region.

3.1. Threshold Segmentation. The threshold segmentation mainly eliminates the background noise. Since the ionosphere echoes from high frequency wave should not exist for less than $60 \mathrm{~km}$ in R-D spectrum, the average power in this area is nearly equal to noise power. Generally, clutter noise ratio is about $20 \sim 35 \mathrm{~dB}$, so let $\delta=P_{N}+20$ to preserve ionosphere clutter, where $\delta$ is the threshold and $P_{N}$ is the noise power.

3.2. Generation of Connected Region. The disadvantage of threshold segmentation is that it ignores the shape characteristics of the ionosphere echoes, which are always displayed as plane or band. Consequently, the next processing is the image segmentation by connected region generation.

The R-D spectrum after the second step still contains sporadic noise and a little sea clutter. The widest connected region is chosen as the ionospheric echoes based on the prior information of height. Then the vertical ionosphere echoes can be obtained.

\section{Modeling HFSWR Ionospheric Backscatter Characteristics}

4.1. Electron Density Estimations by Radar Equation. The classical radar equation for a monostatic HFSWR is defined as

$$
P_{r}=\frac{P_{t} G_{t} G_{r} \lambda^{2}}{(4 \pi)^{3} R^{4} L_{s}} \sigma,
$$

where $P_{r}$ is the received power; $P_{t}$ is the transmitted power; $G_{t}$ is the transmitter antenna gain; $G_{r}$ is the receiver antenna gain; $\sigma$ is the radar cross section (RCS); $\lambda$ is the radar wavelength; $R$ is the target range; $L_{s}$ is the system loss.

The ionospheric scatters cannot be modeled as a single point scatter but distributed scattering from a three-dimensional volume. Thus, a more appropriate form of the generalized radar equation can be given by

$$
\begin{aligned}
& P_{r} \\
& \quad=\frac{P_{t} \lambda^{2}}{(4 \pi)^{3} L_{s}} \int_{\Delta V(R, \theta, \phi)} \frac{G_{t}(\theta, \phi) G_{r}(\theta, \phi)}{R^{4}} d \sigma(R, \theta, \phi),
\end{aligned}
$$

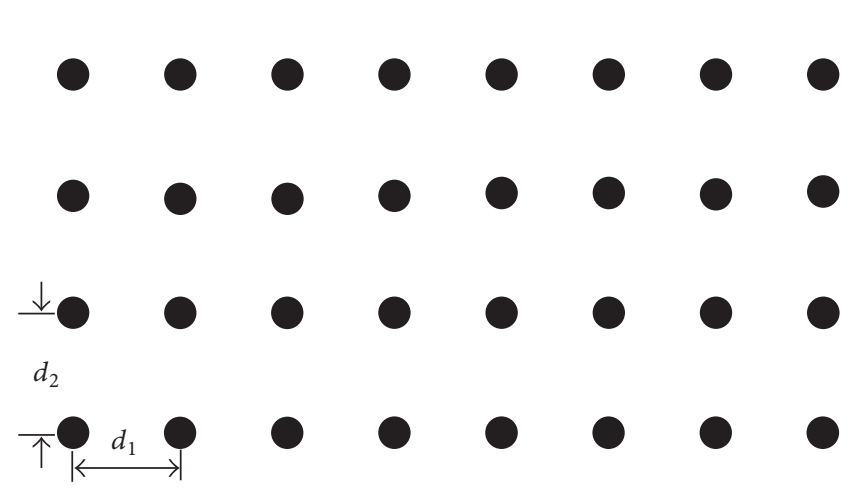

FIGURE 4: Receiving antenna arrays.

where $\Delta V(R, \theta, \phi)$ is the volume of the resolution cell at nominal coordinates $(R, \theta, \phi)$, and

$$
\Delta V(R, \theta, \phi)=\frac{\pi}{4} R^{2} \Delta R \theta_{3} \phi_{3} \approx R^{2} \Delta R \theta_{3} \phi_{3},
$$

where $\Delta R$ is the range resolution and $\theta_{3}, \phi_{3}$ are the $3 \mathrm{~dB}$ beam widths in azimuth and elevation. Considering the attenuation of electromagnetic wave propagation in ionosphere and using approximation, we can reduce (2) to the range equation for ionosphere scatters:

$$
P_{r}=\frac{P_{t} \lambda^{2} G_{t} G_{r} \eta V}{(4 \pi)^{3} R^{4} L_{s} L_{p}}
$$

where $\eta$ is the reflectivity of effective scatter volume (ESV) which is formed by the intersection of radar beam with ionosphere. $V$ is the volume of ionospheric scatter. $L_{p}$ is the attenuation of electromagnetic wave propagation in ionosphere. $\eta$ and $L_{p}$ are discussed in detail as follows.

4.1.1. ESV Reflectivity. It is convincing that the ionospheric echoes of HFSWR mainly occur from coherent scattering between electromagnetic wave and irregularities caused by plasma instabilities. According to the Bragg scatter conditions for monostatic backscatter [15],

$$
\lambda_{\text {irr }}=\frac{\lambda_{\text {radar }}}{2},
$$

where $\lambda_{\text {irr }}$ is the scale size of ionosphere irregularities, which means that the scale size of irregularities between 5 and $50 \mathrm{~m}$ can be observed by HFSWR. For the magnetic plasma in the ionosphere region, irregularities at these scale sizes are highly anisotropic and aligned with the geomagnetic field lines. The reflectivity of effective scatter volume irregularities can be expressed as follows [16]:

$$
\eta \propto \overline{\Delta N^{2}} \exp \left\{-2 k^{2}\left(l_{\|}^{2} \psi^{2}+l_{\perp}^{2}\right)\right\},
$$

where $\overline{\Delta N^{2}}$ is the average level of the electron density fluctuations, $k$ is the radar wave-vector in the medium, and $l_{\|, \perp}$ is the scale size of irregularities along and across the external magnetic field $\overrightarrow{\mathbf{B}}$, respectively. $\psi$ is the aspect angle between $k$ and $\overrightarrow{\mathbf{B}}$. Equation (6) is based on the assumption 


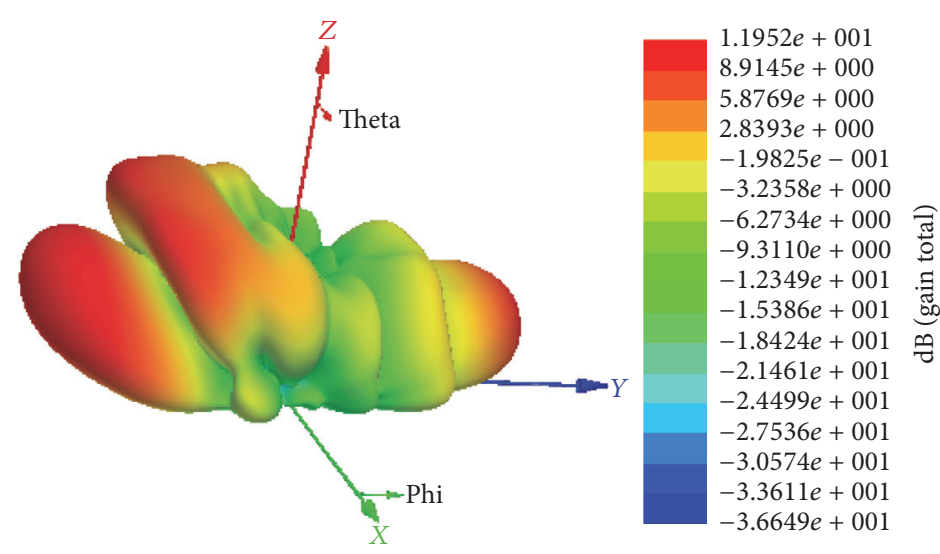

(a) 3D pattern of transmitting antenna at $9 \mathrm{MHz}$

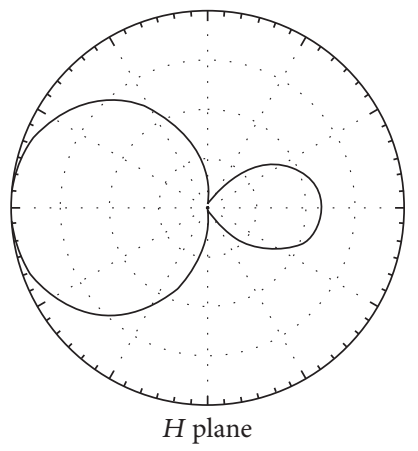

(b) $3 \mathrm{MHz}$

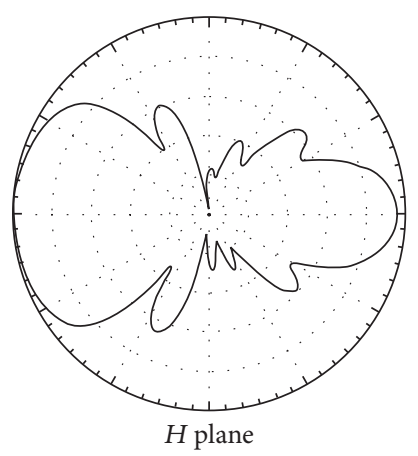

(d) $9 \mathrm{MHz}$
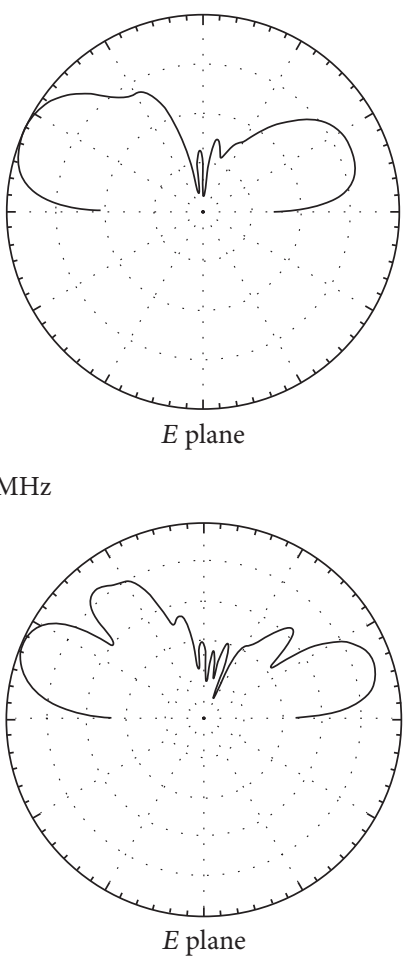
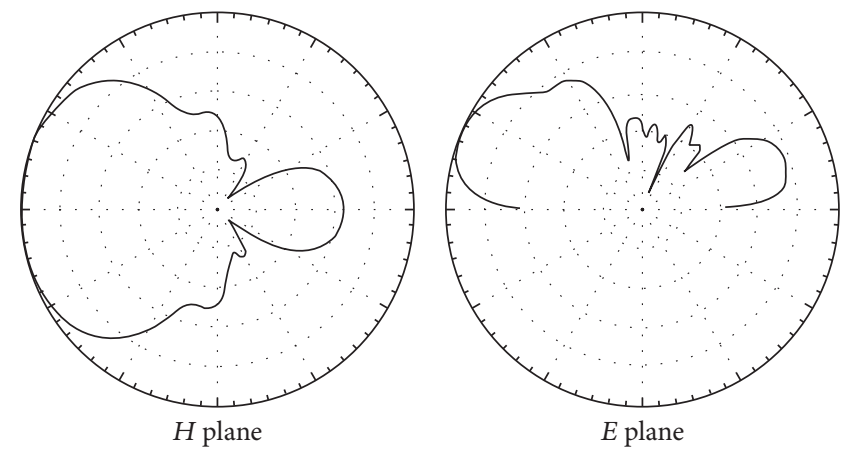

(c) $6 \mathrm{MHz}$
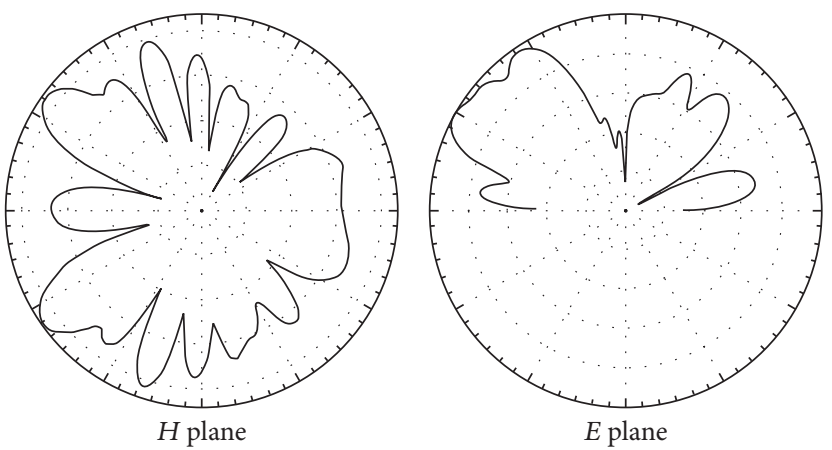

(e) $15 \mathrm{MHz}$

Figure 5: Pattern of log periodic dipole transmitting antenna. The maximum length is $12.5 \mathrm{~m}$. The minimum length is $2.5 \mathrm{~m}$. The copper ground screen is $100 \mathrm{~m}^{2}$. The diameter of grid is $10 \mathrm{~cm}$.

that $l_{\|} \gg l_{\perp}$ and $k l_{\|} \gg 1$ so that $\eta$ exhibits peak when $\psi=0^{\circ}$, namely, $\vec{k} \perp \overrightarrow{\mathbf{B}}$. To simplify (6), we make another assumption: $\overline{\Delta N^{2}} \propto N^{2}$. That implies that magnitude of electron density fluctuations with fixed Bragg scale size $\lambda_{\text {irr }}$ has linear relationship with the electron density, which is also consistent with experimental results [17]. Assuming that other parameters are constant, (6) reduces to

$$
\eta \propto C N^{2}
$$

4.1.2. The Attenuation of Electromagnetic Wave Propagation in Ionosphere. In the mid-latitude region, attenuation of high frequency wave propagation mainly contains the absorption and additional attenuation [18]:

$$
L_{p}=2 A_{i e}+A_{z}
$$

where $A_{i e}$ is the absorption of $\mathrm{D}$ layer and $A_{z}$ is the additional attenuation. Generally,

$$
A_{i e}=\frac{677.2 I}{\left(f+f_{H}\right)^{1.98}+10.2},
$$

where $I$ is the absorption index:

$$
I=\left(1+0.0037 R_{12}\right)[\cos (0.881 \chi)]^{1.3}
$$




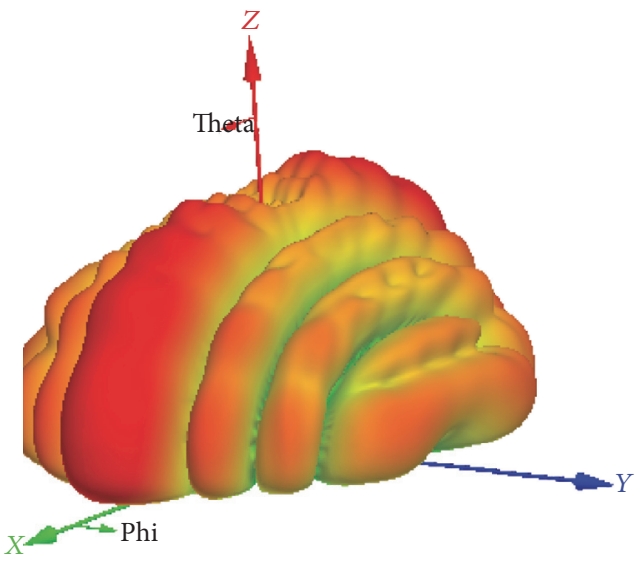

$1.3478 e+00$ $7.2156 e+000$ $9.5288 e-001$

$-5.3099 e+000$

$-1.1573 e+001$

$-1.7835 e+001$

$-2.4098 e+001$

$-3.0361 e+001$

$-3.6624 e+001$

$-4.2886 e+001$ 可

$-4.9149 e+001 \vartheta$

$-5.5412 e+001$

$-6.1675 e+001$

$-6.7937 e+001$

$-7.4200 e+001$

$-8.0463 e+001$

$-8.6726 e+001$

(a) $3 \mathrm{D}$ pattern of receiving arrays at $9 \mathrm{MHz}$

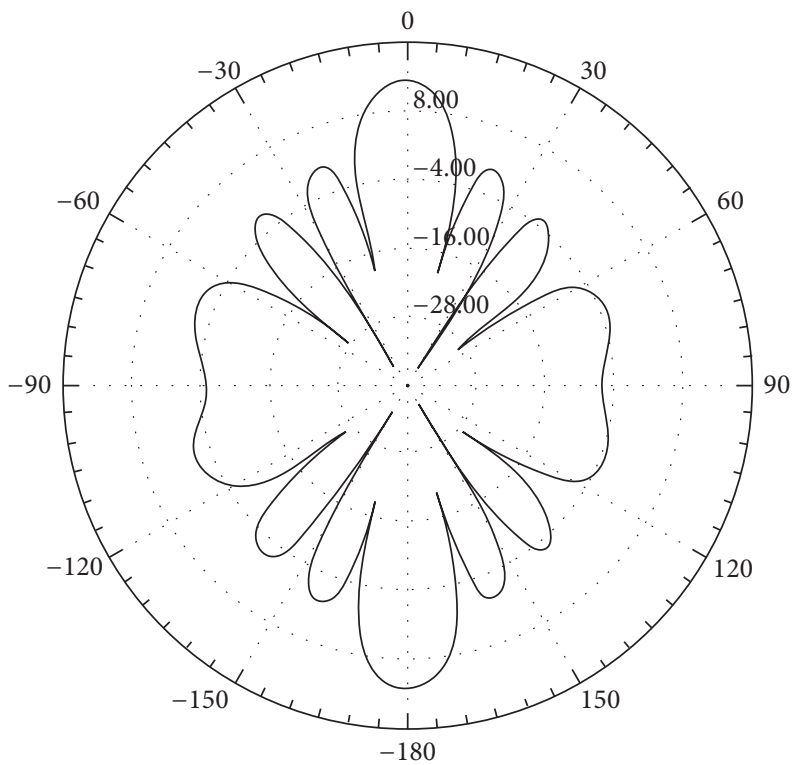

(b) $H$ plane

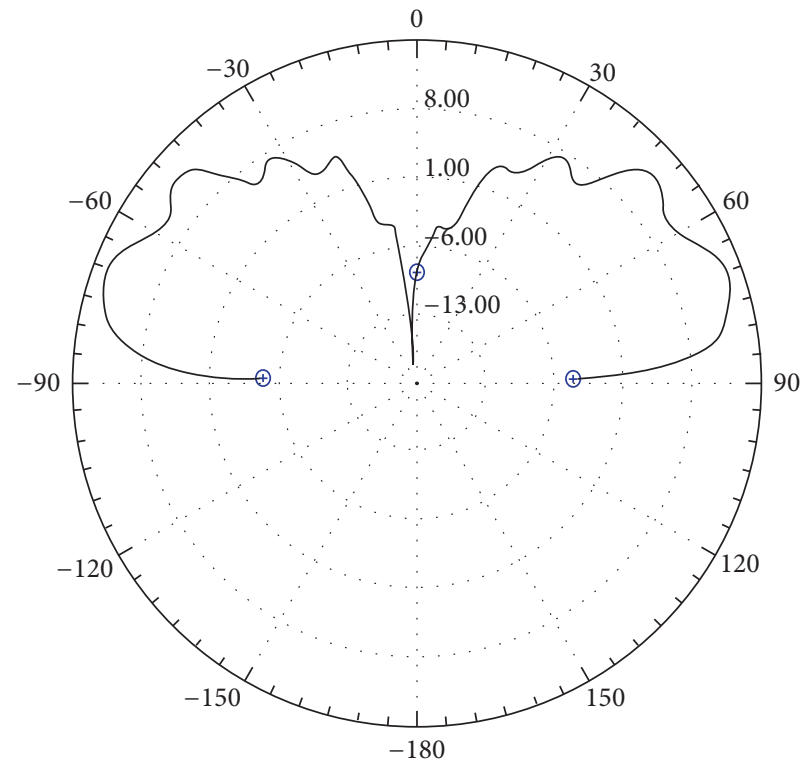

(c) E plane

Figure 6: Pattern of vertically polarized antenna receiving arrays at $9 \mathrm{MHz}$. The element length is $6.5 \mathrm{~m} . d_{1}=14.5 \mathrm{~m}$ and $d_{2}=8 \mathrm{~m}$. The copper ground screen is about $100 \mathrm{~m}^{2}$. The diameter of grid is $10 \mathrm{~cm}$.

where $R_{12}$ is the mean value of sunspot in the year. $\chi$ is the local solar zenith angle.

Considering actual impact such as polarization coupling attenuation, ionospheric irregularities, focus, and defocus, usually $A_{z}=9.9 \mathrm{~dB}$. So the electron density can be obtained by

$$
N_{e}=\sqrt{\frac{P_{r} R^{4} L_{s} L_{p}}{C P_{t} \lambda^{2} G_{t} G_{r} V}} .
$$

4.2. Plasma Frequency Estimations from HFSWR. According to the Appleton-Hartree formula, the plasma frequency can be expressed as

$$
f_{p} \simeq \sqrt{80.6 N_{e}}
$$

when

$$
\begin{aligned}
\frac{v}{f_{0}} \ll 1, \\
\frac{f_{B}^{2}}{f_{0}^{2}} \ll 1
\end{aligned}
$$

holds, where $v$ is the ionospheric electron collision frequency and $f_{B}$ is the ionospheric electron cyclotron frequency. For the operating frequency of HFSWR $f_{0} \simeq 3-15 \mathrm{MHz}$, those conditions are easy to be satisfied in the whole $\mathrm{E}$ and $\mathrm{F}$ layer.

4.3. Irregularities Drift Velocity Estimations from HFSWR. The velocity of irregularities caused by plasma instabilities can be obtained from the Doppler shift of the HFSWR coherent backscatter signal:

$$
v_{\text {irr }}=-\frac{\lambda_{0}}{2} f_{d}
$$



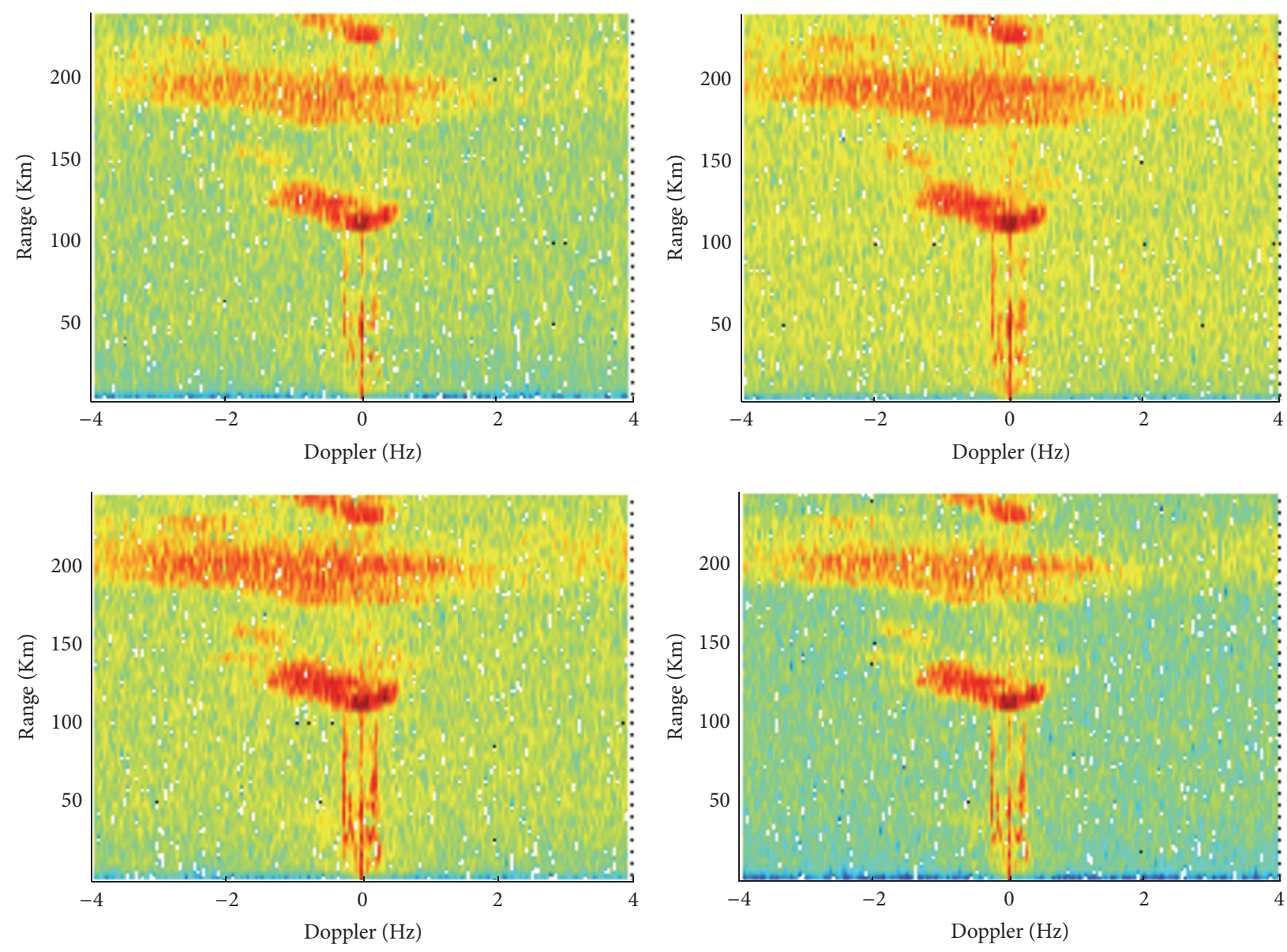

FIGURE 7: R-D spectrums.

where $v_{\text {irr }}$ is the HFSWR line-of-sight velocity of irregularities, which drift at the $\overrightarrow{\mathbf{E}} \times \overrightarrow{\mathbf{B}}$ direction in the ionospheric region. $\lambda_{0}$ is the HFSWR wavelength and $f_{d}$ is the Doppler shift. For a given range bin of ionosphere echoes, the mean drift velocity of plasma is the first moment of Doppler spectrum:

$$
\overline{v_{\mathrm{irr}}}=\frac{\int f_{d} s\left(f_{d}\right) d f_{d}}{\int s\left(f_{d}\right) d f_{d}},
$$

where $s\left(f_{d}\right)$ is the power spectral density function of $f_{d}$.

\section{Experiments and Analysis}

The HFSWR location is as follows:

longitude: $122.1^{\circ}$ and latitude: $37.5^{\circ}$.

The main system parameters are as follows:

Pulse repetition frequency: $T_{p}=4 \mathrm{~ms}$.

Sweep period: $T_{\mathrm{sw}}=32 \times T_{p}=128 \mathrm{~ms}$.

Coherent processing interval: $T_{A}=T_{\mathrm{sw}} \times 256=$ $32.8 \mathrm{~s}$.

Bandwidth: $B_{w}=30 \mathrm{kHz}$.
Range resolution: $\Delta R=5 \mathrm{~km}$.

Peak transmitting power: $P_{t}=2 \mathrm{~kW}$.

Beam direction (1-7): $-48^{\circ},-32^{\circ},-16^{\circ}, 0^{\circ}, 16^{\circ}, 32^{\circ}$, and $48^{\circ}$.

The experiments were conducted at night, morning, and afternoon, respectively. Figure 7 is the R-D spectrum of the beams with operating frequency $5.5 \mathrm{MHz}$ at Beijing time 2014.5.23.20:31, local time 2014.5.23.20:41.

Generally the height of E layer is about $90-150 \mathrm{~km}$ above the surface of earth while $150-500 \mathrm{~km}$ corresponds to $\mathrm{F}$ layer. There are three parts of ionosphere echoes appearing at about $120 \mathrm{~km}, 200 \mathrm{~km}$, and $240 \mathrm{~km}$, with Doppler shift between $[-1,0] \mathrm{Hz},[-4,1] \mathrm{Hz}$, and $[-1,0] \mathrm{Hz}$, respectively. The former echoes occupying a few range bins may come from the specular reflection of Es layer, since E layer usually disappears at night. The middle echoes may come from spread of $\mathrm{F}$ layer since it occupied about 8 range bins $(40 \mathrm{~km})$ and a large number of Doppler bins. The last echoes probably are the Es layer second-bounce since they have the same Doppler distribution and the radar operating frequency is much lower than $f_{0} \mathrm{~F} 2$.

After threshold segmentation and connected region generation, the vertical ionosphere echoes are shown in Figure 8. Although the peak power of Es layer echoes is higher than 
TABLE 1: Experimental results compared with IRI2012.

\begin{tabular}{ccccccc}
\hline Time & $\begin{array}{c}\text { Radar frequency } \\
f / \mathrm{MHz}\end{array}$ & $\begin{array}{c}\text { Ionosphere height } \\
h / \mathrm{km}\end{array}$ & $\begin{array}{c}\text { Electron density } \\
N_{e} / \mathrm{m}^{-3}\end{array}$ & $\begin{array}{c}\text { IRI2012 estimation } \\
N_{e} / \mathrm{m}^{-3}\end{array}$ & $\begin{array}{c}\text { Plasma frequency } \\
f_{p} / \mathrm{MHz}\end{array}$ & \multicolumn{3}{c}{$\begin{array}{c}\text { IRI2012 estimation } \\
f_{p} / \mathrm{MHz}\end{array}$} & \multicolumn{2}{c}{ Mean velocity } \\
$v / \mathrm{m} / \mathrm{s}$
\end{tabular}

F layer echoes; the total power is just the opposite. The mean Doppler shifts of ionosphere echoes from Es layer and $\mathrm{F}$ layer are $-0.48 \mathrm{~Hz}$ and $-1.68 \mathrm{~Hz}$, respectively. So the mean drift velocities of irregularities are $-13 \mathrm{~m} / \mathrm{s}$ and $-45 \mathrm{~m} / \mathrm{s}$, respectively. The electron density and plasma frequency corresponding to ionospheric scattering volume also can be estimated by (11) and (12), respectively. Table 1 shows the experimental results compared with that of IRI2012 in the order of time: May 23 evening, 24 afternoon, and 25 morning 2014.

Table 1 shows that the electron density from (11) roughly has the same order of magnitude as IRI. The difference between estimation values and IRI may be caused by the assumption of constant in (6). The estimated values of the day accord with the IRI better than that of the night. The observational data at night has always been different from IRI. For example, there are so many intense ionospheric echoes from E layer for HFSWR while E layer disappeared in IRI. And those echoes always tend to last a few hours and occupy several range bins which is different from Es layer. Therefore Jiang et al. refer to the new ionospheric structure as spread E layer [11].

\section{Conclusions}

The main purpose of this paper is to reveal the physical mechanism of the ionospheric clutter for HFSWR. Unlike most papers concentrating on ionospheric clutter suppression, this paper tries to preform sounding ionosphere by the received ionospheric clutter. Therefore the HFSWR can not only detect target in over-the-horizon detection and ocean remote sensing but also perform sounding the ionosphere in the range of radar coverage. Firstly, the pattern of transmitting antenna and receiving antenna is simulated by HFSS so that we can know the spatial distribution of the ionosphere. After the pretreatment of ionospheric echoes we get the vertical HFSWR ionosphere echoes. Then we discussed the coherent scattering mechanism and established the mathematical modeling of the receiving power and electron density of irregularities. So the electron density of FAIs created by plasma instabilities in the corresponding ionosphere region can be obtained by the coherent backscattered radar equation

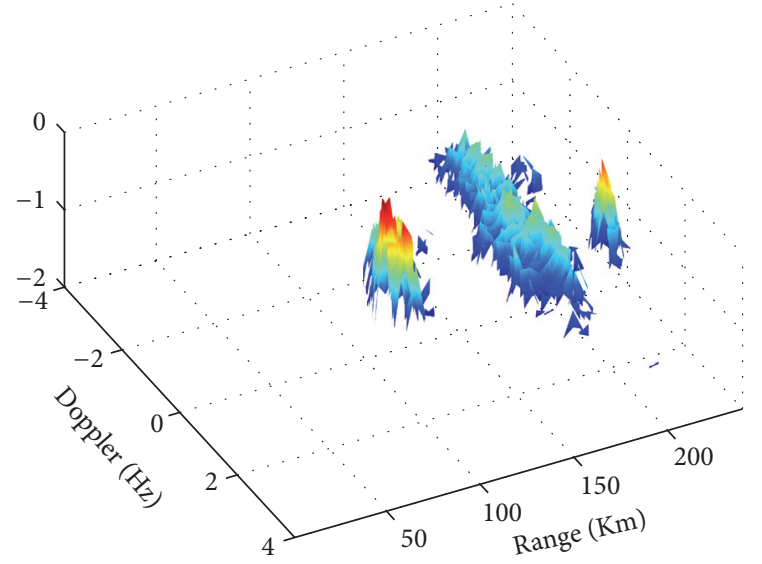

FIgURE 8: The vertical ionospheric echoes.

while plasma frequency and drift velocity can be obtained at the same time. Most of the experimental results are roughly consistent with IRI2012 except data in the evening.

The function of HFSWR looks like ionosonde in this paper. In fact, this is just the first step of our future works. Next we will retrieve the ionospheric parameters of the whole HFSWR coverage region which may have over ten thousand cubic kilometers and be hardly detected by other ionosphere soundings. The ionospheric information will be very beneficial to shortwave communication, frequency selection for high frequency sky wave radar and HFSWR, and the relationship between ionosphere and sea state.

\section{Competing Interests}

The authors declare that they have no competing interests.

\section{Acknowledgments}

The authors would like to express their sincere thanks to the National Natural Science Foundation of China (Grants nos. 61171188 and 61571159) and members of the school of Electronics and Information Engineering, Research Center, Harbin Institute of Technology, for technical support. 


\section{References}

[1] E. D. R. Shearman, "Propagation and scattering in MF/HF ground wave radar," IEE Proceedings, Part F: Communications, Radar and Signal Processing, vol. 130, no. 7, pp. 579-590, 1983.

[2] D. Barrick, "History, present status, and future directions of HF surface-wave radars in the U.S," in Proceedings of the International Conference on Radar (RADAR '03), Adelaide, Australia, September 2003.

[3] H. C. Chan and E. K. L. Hung, "An investigation in interference suppression for HF surface wave radar," Tech. Rep. 2000-028, Defense R\&D Canada, Ottawa, Canada, 1999.

[4] L. Sevgi, A. Ponsford, and H. C. Chan, "An integrated maritime surveillance system based on high-frequency surface-wave radars, Part 1: theoretical background and numerical simulations," IEEE Antennas and Propagation Magazine, vol. 43, no. 4, pp. 28-43, 2001.

[5] A. M. Ponsford, L. Sevgi, and H. C. Chan, "An integrated maritime surveillance system based on high-frequency surface-wave radar, part II: operational status and system performance," IEEE Antennas and Propagation Magazine, vol. 43, no. 5, pp. 52-63, 2001.

[6] X. Zhang, Q. Yang, and W. Deng, "Weak target detection within the nonhomogeneous ionospheric clutter background of HFSWR based on STAP," International Journal of Antennas and Propagation, vol. 2013, Article ID 382516, 11 pages, 2013.

[7] Y. Li, Y. Wei, R. Xu, Z. Wang, and T. Chu, "An ionospheric Es layer clutter model and suppression in HF surfacewave Radar," International Journal of Antennas and Propagation, vol. 2013, Article ID 320645, 18 pages, 2013.

[8] H. C. Chan, "Characterization of ionospheric clutter in HF surface-wave radar," Tech. Rep. 2003-114, Defence R\&D, Ottawa, Canada, 2003.

[9] S. Shang, N. Zhang, and Y. Li, "Ionospheric clutter statistical properties in HFSWR," Chinese Journal of Radio Science, vol. 26, no. 3, pp. 521-527, 2011.

[10] X. Wan, X. Xiong, F. Cheng, and H. Ke, "Experimental investigation of directional characteristics for ionospheric clutter in HF surface wave radar," IET Radar, Sonar \& Navigation, vol. 1, no. 2, pp. 124-130, 2007.

[11] W. Jiang, W. Deng, and J. Shi, "Characteristic study of ionospheric clutter in high frequency over the horizon surface wave radar," in Proceedings of the IEEE China Youth Conference on Information and Communications Technology, pp. 154-157, Beijing, China, 2009.

[12] D. Bilitza, D. Altadill, Y. Zhang et al., "The international reference ionosphere 2012-a model of international collaboration," Journal of Space Weather \& Space Climate, vol. 4, article A07, 2014.

[13] H. Zhou, B. Wen, and S. Wu, "Ionosphere probing with a high frequency surface wave radar," Progress In Electromagnetics Research C, vol. 20, pp. 203-214, 2011.

[14] H. Gao, G. Li, Y. Li, Z. Yang, and X. Wu, "Ionospheric effect of HF surface wave over-the-horizon radar," Radio Science, vol. 41, no. 6, Article ID RS6S36, 2006.

[15] K. Schlegel, "Coherent backscatter from ionospheric E-region plasma irregularities," Journal of Atmospheric and Terrestrial Physics, vol. 58, no. 8-9, pp. 933-941, 1996.

[16] P. V. Ponomarenko, J.-P. St-Maurice, C. L. Waters, R. G. Gillies, and A. V. Koustov, "Refractive index effects on the scatter volume location and Doppler velocity estimates of ionospheric
HF backscatter echoes," Annales Geophysicae, vol. 27, no. 11, pp. 4207-4219, 2009.

[17] C. Haldoupis, E. Nielsen, and K. Schlegel, "Dependence of radar auroral scattering cross section on the ambient electron density and the destabilizing electric field," Annals of Geophysics, vol. 8, no. 3, pp. 195-211, 1900.

[18] CCIR Recommendation 252-2, Second CCIR computer-based interim method for estimation sky-wave field strength and transmission loss at frequencies between 2 and $30 \mathrm{MHz}, 1992$. 


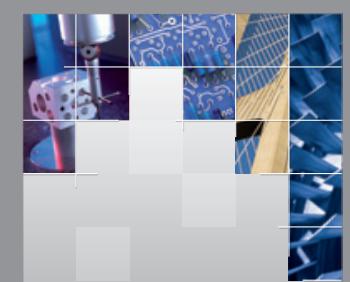

\section{Enfincering}
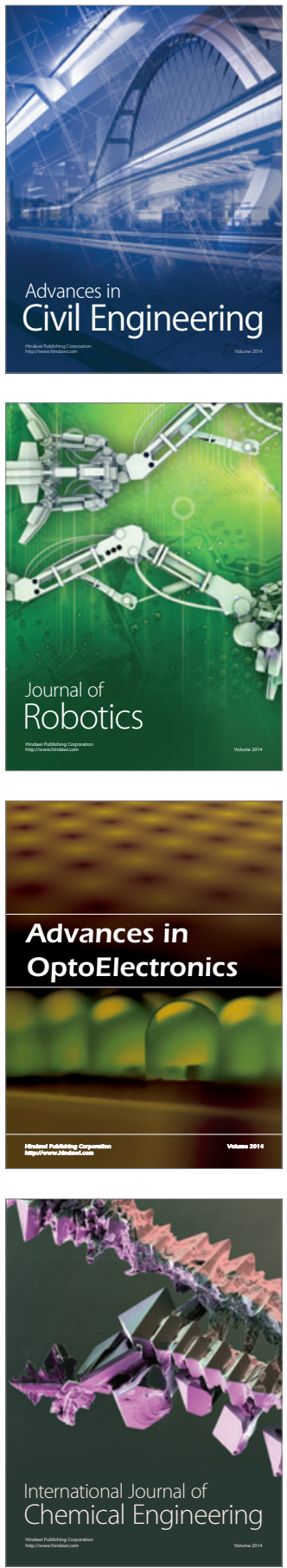

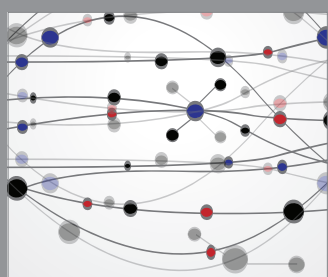

The Scientific World Journal

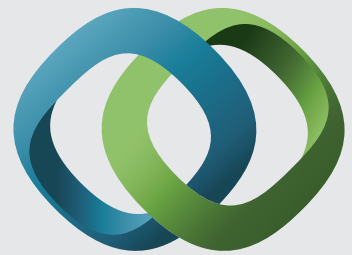

\section{Hindawi}

Submit your manuscripts at

http://www.hindawi.com
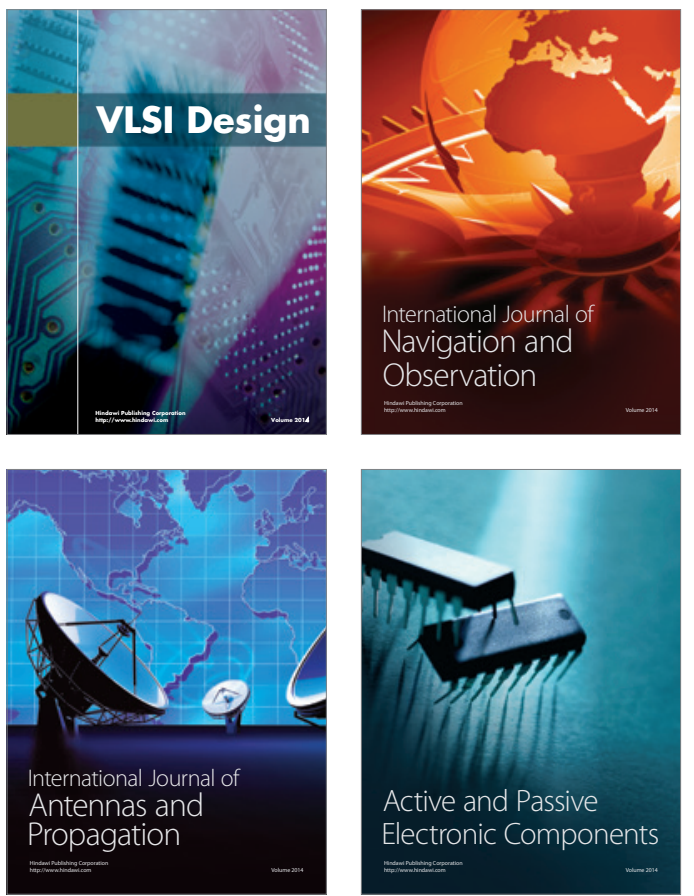
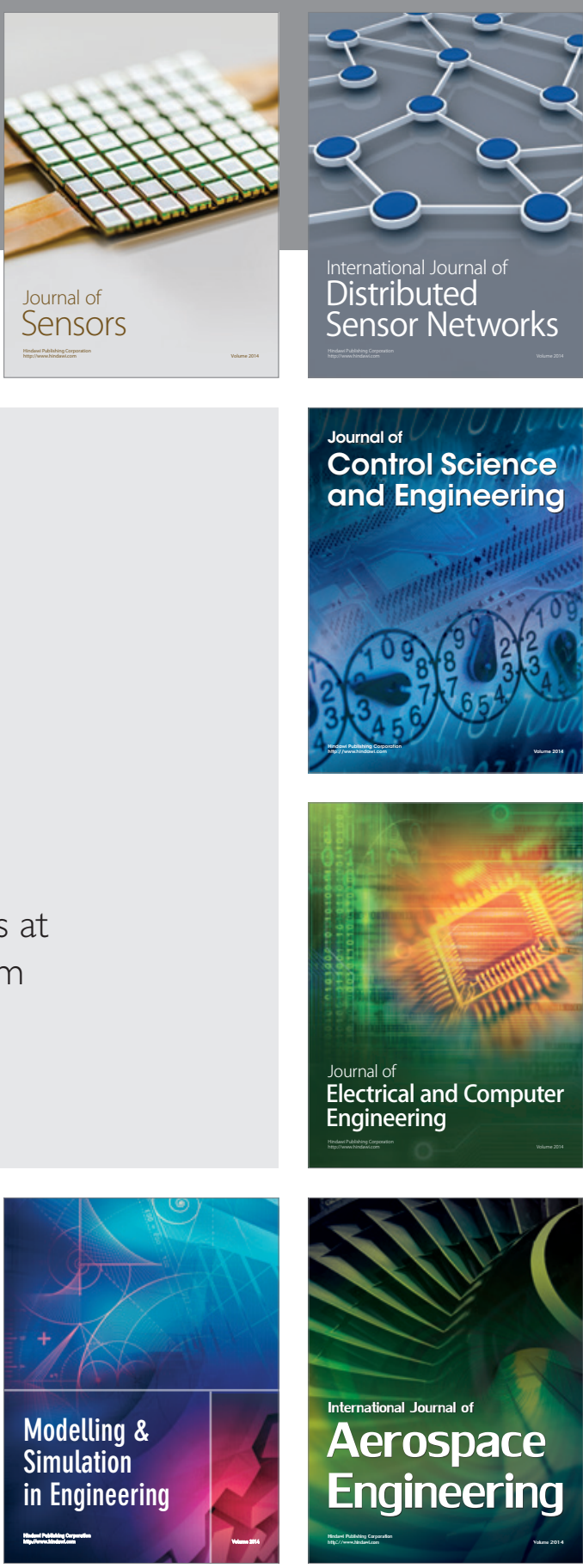

International Journal of

Distributed

Sensor Networks

Journal of

Control Science

and Engineering
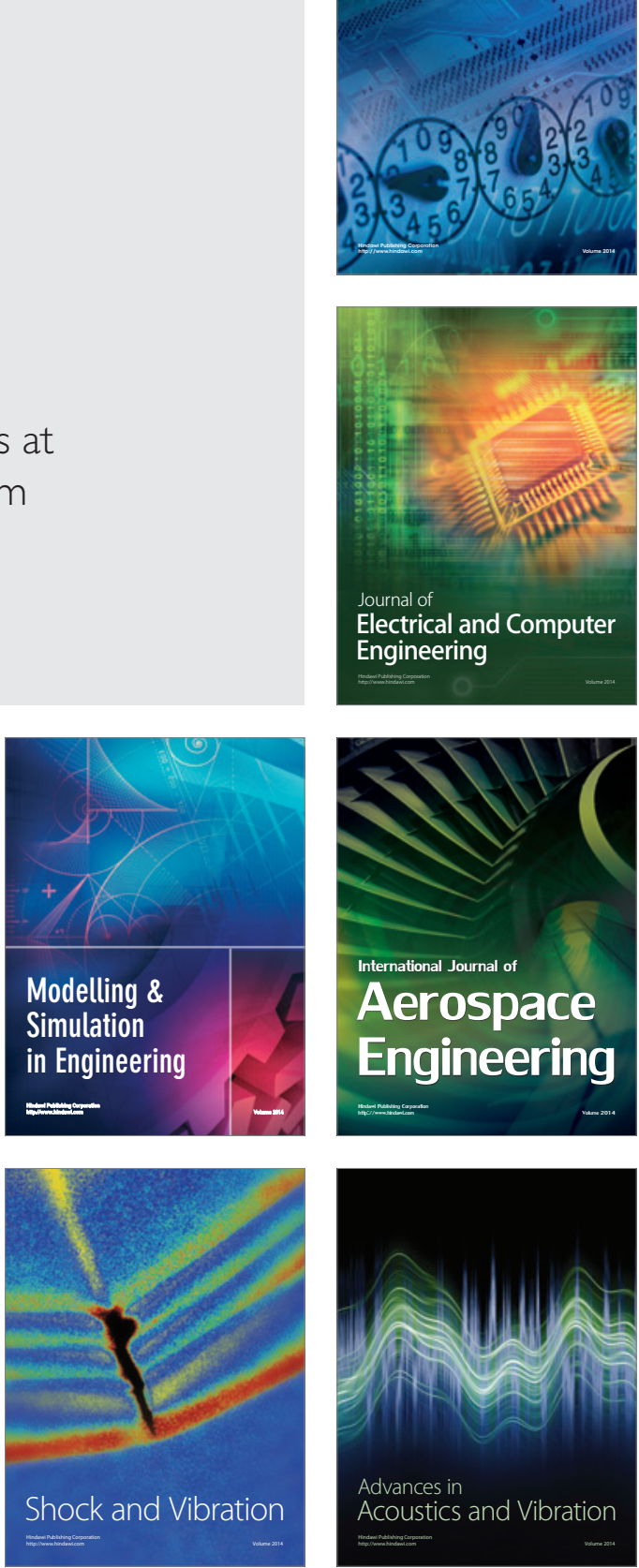